\title{
Relative event localization in uncertain velocity model
}

Oleg V. Poliannikov ${ }^{*, 1}$, Michael Prange ${ }^{2}$, Alison Malcolm ${ }^{1}$, and Hugues Djikpesse ${ }^{2}$

${ }^{1}$ Earth Resources Laboratory, MIT; ${ }^{2}$ Schlumberger-Doll Research

\section{SUMMARY}

We study a problem of localization of an unknown event location relative to previously located events using a single monitoring array in a monitoring well. It has been shown that using the available information about the previously located events for locating new events is advantageous to localizing each event independently. We compare the performance of two previously proposed localization methods, double-difference and interferometry, in varying signal noise and velocity uncertainty, and propose a framework for selecting the optimal method for a given experiment.

\section{INTRODUCTION}

A collection of seismic events excites a signal that is then recorded by receivers in a monitoring well. The problem is to localize these events using the recorded signal. A conventional method of event localization is to localize events individually. For each event, the noisy travel time, $\hat{T}_{j}=T\left(\mathbf{s}, \mathbf{r}_{j}\right)+\varepsilon_{j}, j=$ $1, \ldots, N_{\mathrm{r}}$, is picked at each of $N_{\mathrm{r}}$ receivers. Here $T\left(\mathbf{s}, \mathbf{r}_{j}\right)$ is the predicted travel time from event $\mathbf{s}$ to receiver $\mathbf{r}_{j}$ computed by raytracing the assumed velocity model $V ; \varepsilon_{j}$ is independent Gaussian noise, and $\varepsilon_{j} \sim \mathscr{N}\left(0, \sigma_{j}^{2}\right)$.

In practice, we may need to localize many seismic events. Instead of localizing the events one by one it is beneficial to use the available information about the previously located events in order to localize subsequent events. Assume that we have already localized $N_{\mathrm{s}}$ events: $\mathbf{s}_{1}, \ldots, \mathbf{s}_{N_{\mathrm{s}}}$. Then we can use the original waveforms to compute cross-correlations of direct arrivals from events $\mathbf{s}_{i}$ with that from event $\mathbf{s}$. The goal is to localize an unknown event $\mathbf{s}$. In order to use information from the already localized events, we use the original waveforms to compute cross-correlations of direct arrivals from events $\mathbf{s}_{i}$ with that from event $\mathbf{s}$ and pick lags: $\hat{\tau}_{i, j}=\tau\left(\mathbf{s}_{i}, \mathbf{s}, \mathbf{r}_{j}\right)+\eta_{i, j}$, where $\tau\left(\mathbf{s}_{i}, \mathbf{s}, \mathbf{r}_{j}\right)=T\left(\mathbf{s}, \mathbf{r}_{j}\right)-T\left(\mathbf{s}_{i}, \mathbf{r}_{j}\right)$ is the projected correlogram moveout in the assumed velocity model, and $\eta_{i, j}$ is independent Gaussian noise given by $\eta_{i, j} \sim \mathscr{N}\left(0, \zeta_{i, j}^{2}\right)$.

We compare two methods of relative localization of the unknown event location $\mathbf{s}$ using the already known event locations $\mathbf{s}_{1}, \ldots, \mathbf{s}_{N_{\mathrm{s}}}$. Both are based on fitting predicted moveouts of correlogram events into observed correlograms but they use different fitting norms. In what follows we will describe both methods, compare their performance and discuss their relative strengths and weaknesses.

\section{DOUBLE-DIFFERENCE LOCALIZATION}

The first method of relative localization is based on fitting the predicted lag moveouts to the observed lag picks using $\ell_{2}$ - norm. The resulting probability density function (pdf) of the event location has the form:

$$
\begin{aligned}
p_{\mathrm{dd}}\left(\mathbf{s} \mid \mathbf{s}_{1}, \ldots, \mathbf{s}_{N_{\mathrm{s}}}, V\right)=\frac{1}{(2 \pi)^{\frac{N_{\mathrm{r}} N_{\mathrm{s}}}{2}} \prod_{i=1}^{N_{\mathrm{s}}} \prod_{j=1}^{N_{\mathrm{r}}} \zeta_{i, j}} \\
\quad \times \exp \left[-\frac{1}{2} \sum_{i=1}^{N_{\mathrm{s}}} \sum_{j=1}^{N_{\mathrm{r}}}\left(\frac{\hat{\tau}_{i, j}-\tau\left(\mathbf{s}_{i}, \mathbf{s}, \mathbf{r}_{j} \mid V\right)}{\zeta_{i, j}}\right)^{2}\right],
\end{aligned}
$$

where we emphasize the dependence of the final distribution on the assumed velocity model. If $V$ is assumed to be a sample from a family of velocity models $\mathscr{V}$ then the marginal distribution is computed by averaging over $\mathscr{V}$ :

$$
p_{\mathrm{dd}}\left(\mathbf{s} \mid \mathbf{s}_{1}, \ldots, \mathbf{s}_{N_{\mathrm{s}}}\right)=\sum_{V \in \mathscr{V}} p_{\mathrm{dd}}\left(\mathbf{s} \mid \mathbf{s}_{1}, \ldots, \mathbf{s}_{N_{\mathrm{s}}}, V\right) p(V)
$$

\section{INTERFEROMETRIC LOCALIZATION}

The second localization method is based on interferometry. For each event, we perform a stationary phase analysis of the correlogram event moveout and fit the lag and the stationary time at the location of the stationary receiver only. The resulting location estimator has a pdf written as

$$
\begin{aligned}
p_{\text {int }}\left(\mathbf{s} \mid \mathbf{s}_{1}, \ldots, \mathbf{s}_{N_{\mathrm{s}}}, V\right)=\frac{1}{(2 \pi)^{N_{\mathrm{s}}} \prod_{i=1}^{N_{\mathrm{s}}} 2 \zeta_{i, *}^{2}} \\
\times \exp \left[-\frac{1}{2} \sum_{i=1}^{N_{\mathrm{s}}}\left(\frac{\hat{\tau}_{i, *}-\tau\left(\mathbf{s}_{i}, \mathbf{s}, \mathbf{r}_{i}^{*} \mid V\right)}{\zeta_{i, *}}\right)^{2}\right] \\
\times \exp \left[-\frac{1}{2} \sum_{i=1}^{N_{\mathrm{s}}}\left(\frac{\partial_{\mathbf{r}} \tau\left(\mathbf{s}_{i}, \mathbf{s}, \mathbf{r}_{i, *} \mid V\right)}{2 \zeta_{i, *}}\right)^{2}\right]
\end{aligned}
$$

where $\partial_{\mathbf{r}} \tau\left(\mathbf{s}_{i}, \mathbf{s}, \mathbf{r}_{i, *} \mid V\right)=0$, and $\hat{\tau}_{i, *}=\tau\left(\mathbf{s}_{i}, \mathbf{s}, \mathbf{r}_{i, *} \mid V\right)$, and

$$
p_{\text {int }}\left(\mathbf{s} \mid \mathbf{s}_{1}, \ldots, \mathbf{s}_{N_{\mathrm{s}}}\right)=\sum_{V \in \mathscr{V}} p_{\text {int }}\left(\mathbf{s} \mid \mathbf{s}_{1}, \ldots, \mathbf{s}_{N_{\mathrm{s}}}, V\right) p(V),
$$

\section{COMPARISON OF THE TWO METHODS}

It is found that the performance of each localization method depends on the strength of the noise in the recorded signal as well as the uncertainty in the assumed velocity model. We show that the double-difference method combats the signal noise much better due to the averaging over a larger number of data points. The interferometric method is superior where the main source of error is the velocity uncertainty between the events and the monitoring array. The optimal method is one that produces the smallest uncertainty region for the same confidence level. 
http://dx.doi.org/10.1190/segam2012-0154.1

\section{EDITED REFERENCES}

Note: This reference list is a copy-edited version of the reference list submitted by the author. Reference lists for the 2012 SEG Technical Program Expanded Abstracts have been copy edited so that references provided with the online metadata for each paper will achieve a high degree of linking to cited sources that appear on the Web.

\section{REFERENCES}

None 